\title{
Ligneous Cellulitis Following Spontaneous Vaginal Delivery
}

\author{
J.F. Wyatt and G.R.G. Monif* \\ Department of Obstetrics and Gynecology, Creighton University School of Medicine, Omaha, NE
}

ligneous cellulitis; Streptococcus milleri; thrombophlebitis

$\mathrm{L}$ igneous cellulitis is a rare chronic indolent cellulitis that often includes the parametrium or retroperitoneal tissues. ${ }^{1-5}$ Clinically, at a time reasonably distant from surgery, the patient may report fatigue, anorexia, vague pelvic discomfort, and/or weight loss. She may or may not be febrile. Not infrequently, abnormal or prolonged uterine bleeding may be the main reason the patient seeks medical care. Bimanual examination reveals fixation of the pelvis. The pelvic floor has a characteristic woody, hard, smooth texture.

The purpose of this paper is to report a case of early ligneous cellulitis that developed after spontaneous vaginal delivery.

\section{CASE REPORT}

The patient is a 25 -year-old female, gravida 2, para 1-1-0-1, who presented at six weeks' postpartum with a five-day history of fever, headache, nausea, vomiting, chills and diarrhea, which she attributed to the flu. Six weeks previously, she had undergone an assisted, uncomplicated vaginal delivery at 37 weeks' gestation at Saint Joseph Hospital in Omaha, Nebraska. Her prenatal course was notable for the finding of a positive fluorescent antinuclear antibody screen of a speckled pattern less than $1-40$ dilution and an antinuclear antibody titer of 1-80. The patient's prothrombin time was 13.2, and partial thromboplastin time (P'TT) was 32.5 with a leuco anticoagulant dRVVT of 23.8. She had a low positive anticardiolipin panel with a quantitative immunoglobulin $G$ antibody of 12 and quantitative immunoglobulin $\mathrm{M}$ antibody of 4 . Platelet count was 327,000 at baseline. The patient is $\mathrm{O}$ positive. The antibody screen, rubella serology, syphyllis screen, DNA probes for Neisseria gonorrhoeae and Chlamydia trachomatis, and hepatitis surface B antigen assay, as well as vaginal cultures for group B streptococci were all negative. She had an uncomplicated prenatal course with normal blood pressure and was uniformly progressing with fundal growth. Serial real-time ultrasound assessment showed appropriate and progressive fetal growth, normal amniotic fluid volume and indices, and a normal-appearing placenta. During labor, the patient received an epidural anesthetic. The first stage of labor lasted 11 hours and 15 minutes, the second stage one hour and nine minutes, and her third stage lasted two minutes with placental separation. Estimated blood loss was $250 \mathrm{~mL}$. The patient did not receive perineal injection or local anesthesia. The right paramedian episiotomy was utilized for delivery, and there was no extension.

The patient returned for postpartum assessment one week after delivery at which time a vaginal examination was performed that demonstrated nor-

*Correspondence to: Gilles R.G. Monif, MD, Department of Obstetrics and Gynecology, Creighton University School of Medicine, 601 North 30th Street, Omaha, NE 68131. 


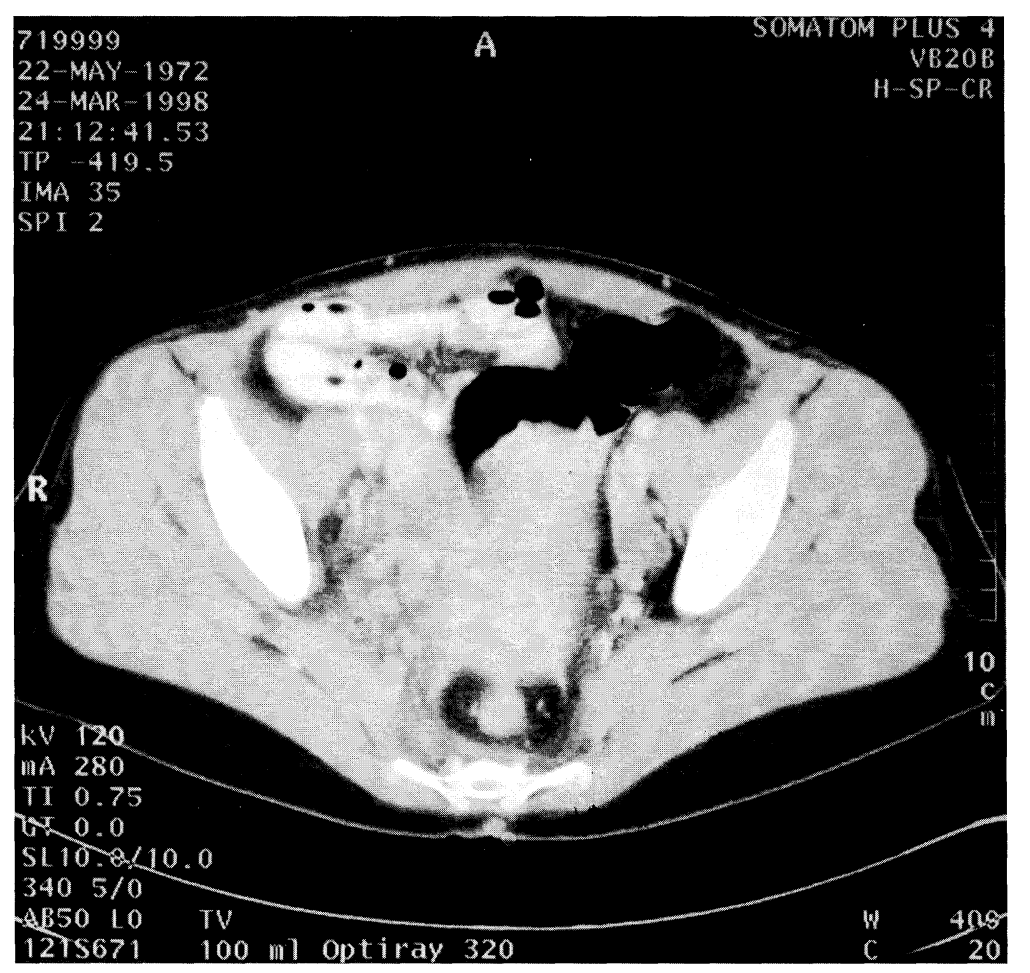

Fig. I. Computed tomography demonstrated a nonspecific inflammatory change in the patient's right pelvis. mal episiotomy healing, and no pelvic masses or abnormal tenderness were noted.

On initial examination, the patient's temperature was $102.6^{\circ} \mathrm{F}\left(39.2^{\circ} \mathrm{C}\right)$ orally. The patient appeared ill but was in no acute distress. Physical examination was unremarkable except for a tender, soft, tissue mass involving the right posterior retroperitoneal region. Transvaginal ultrasound indicated a normal-appearing right ovary with no free fluid in the cul-de-sac. The uterus was anteflexed with a 4-mm endometrial stripe. There appeared to be no complex endometrial echogenicity. The left adnexa was unremarkable. A $3 \times 2.5 \times 5$-cm, complex mass was visualized along the right posterior pelvic sidewall coursing cephalad and laterally toward the right. The mass had a "wormy" appearance. The patient was referred for admission to the hospital, at which time she had a temperature of $102^{\circ} \mathrm{F}$, a pulse of 98 , and a respiratory rate of 20 . Physical examination confirmed the previous findings.

Laboratory and X-ray data revealed a white blood cell count of 14,200 with a left shift, Dohle bodies, and toxic granulation. Chest X-ray showed no effusions, infiltrates, or lesions. Electrolytes demonstrated imbalance with a potassium of 2.9, sodium 135, chloride 94, and a $\mathrm{CO}_{2}$ of 25. Blood urea nitrogen was 5 and creatinine 0.9 . Two of three sets of blood cultures taken at admission produced Streptococcus milleri.

Computerized tomography assessment was performed and demonstrated a nonspecific inflammatory change in her right pelvis (Fig. 1). There was no evidence of a pelvic abscess. The elongated region of inflammation was retroperitoneal and did not appear to have an intraperitoneal component or involve the bowel.

In the hospital, the patient was placed on ampillin/sulbactam, gentamicin, and clindamycin. She was heparinized with $10,000 \mathrm{U}$ of heparin as loading dose, then placed on $1,000 \mathrm{U} / \mathrm{h}$. Her temperature rose to $104^{\circ} \mathrm{F}\left(40^{\circ} \mathrm{C}\right)$ within the first 12 hours of admission. Consultation with Infectious Disease/Obstetrics and Gynecology personnel was obtained on the second hospital day. Because of the significant induration, the diagnosis of ligneous cellulitis was added to those of septic thrombophlebitis and sepsis due to $S$. milleri.

With aggressive therapy, the patient's maximum temperatures on days 3,4 , and 5 were $101.3^{\circ} \mathrm{F}$, $99.9^{\circ} \mathrm{F}$, and $99.3^{\circ} \mathrm{F}\left(38.5^{\circ} \mathrm{C}, 37.7^{\circ} \mathrm{C}\right.$, and $\left.37.4^{\circ} \mathrm{C}\right)$, respectively. Thereafter, her index remained at $98.6^{\circ} \mathrm{F}\left(37.0^{\circ} \mathrm{C}\right)$ and below. Her electrolytes were corrected with electrolyte and fluid replacement. The sedimentation rate was $100 \mathrm{~mm} / \mathrm{h}$ initially, peaking at $123 \mathrm{~mm}$, with a final level of $116 \mathrm{~mm}$ at 
the time of discharge from the hospital. The differential showed a shift over the first four days to $25 \%$ bands. The white blood cell count fluctuated between 11,700 and 18,000. Toxic granulation was present the first five days of treatment, normalizing the differential before dismissal. The initial platelet count was 230,000, with levels of 192,000; 239,000; and 297,000 before elevating on the patient's fifth hospital day to 360,000 . Thereafter, there was a progressive thrombocytosis with a platelet count of 548,000 on the patient's seventh hospital day, subsequently stabilizing between 625,000 and 670,000 . The red blood cell, white blood cell, and platelet morphology normalized. Creactive protein at the time of dismissal was 1.4 . On the third day of therapy, two out of three sets of blood cultures produced $S$. milleri with sensitivities to the antibiotics in dose levels being administered. Ampicillin, clindamycin, and gentamicin were substituted for the initial ampicillin/sulbactam therapy. Heparin was aggressively increased, requiring an infusion rate up to $2,100 \mathrm{U} / \mathrm{h}$ to maintain a therapeutic PTT. The heparin was continued until the patient had normalized her temperature index. Serial physical examination revealed no rebound. An initial adynamic ileus resolved with therapy. A Clostridium difficile toxin proved negative. The heparin was discontinued on the patient's seventh day of treatment. Because patient remained afebrile, triple therapy was discontinued, and she was placed on amoxacillin/clavalanate and metronidazole.

In the ambulatory care setting, antibiotic therapy with amoxacillin/clavalanate and metronidazole was continued as long as the C-reative protein remained elevated. Normalization of the Creactive protein determinations coincided with a return to normal levels of the platelet count. Ultrasonographic evaluation of the liver showed no evidence of abscess formation.

\section{DISCUSSION}

Studies in terms of causative agents and pathogenesis and ligneous cellulitis are poorly delineated. Because the patient probably develops phlebothrombosis and resultant septicemic, case identification occurred relatively early in the course of the disease.

$S$. milleri resides primarily in the gastrointestinal tract and functions as both a facultative and obliga- tory anaerobic bacteria. ${ }^{5-7}$ In gynecologic patients, S. milleri has produced tubo-ovarian abscess and spontaneous necrotic cutaneous infections. ${ }^{6-7}$ The bacterium is frequently isolated in patients with active perineal suppurative hidradentitis. Highet et al. reported that its presence is significantly associated with disease activity, and its disappearance significantly correlates with clinical improvement. ${ }^{8}$ In this clinical setting, $S$. milleri frequently cofunctions with anaerobic bacteria or Staphylococcus aureus. When a bacteremia is induced, metastatic hepatic abscesses may ensue.

As was illustrated in this case, some patients with ligneous cellulitis develop septic pelvic thrombophlebitis heralded by spiking temperature, shaking chills, and sometimes pelvic or abdominal pain. ${ }^{1}$ Septic thrombophlebitis requires intravenous administration of heparin and prolonged aggressive antibiotic therapy. Characteristic of this disease entity was the elevated white blood cell count and erythrocyte sedimentation rate in excess of 100 . With chronicity of disease, anemia secondary to infection becomes more prominent.

The marked increase in platelet count observed is characteristic of patients with chronic retroperitoneal inflammation. ${ }^{8}$ When this phenomenon occurs, it becomes an excellent therapeutic titration point. Once the diagnosis is made, the patient should be hospitalized, treated initially with antibiotics with both aerobic gram-positive and anaerobic coverage, and kept on bed rest. Attention should be given to nutrition. Some clinicians have advocated simultaneous administration of cortisone and other anti-inflammatory agents with antibiotics. $^{2-3}$

When the patient's temperature has returned to normal and she is eating well, she may be discharged from the hospital with instructions to continue treatment at home by resting, adhering to a well-balanced diet, and taking an appropriate antibiotic. Within 6-12 weeks, the cellulitis should resolve.

In advanced cases involving the uterus, bimanual examination reveals fixation of the pelvis. The pelvic floor has a characteristic woody-hard, smooth texture. It is not difficult to comprehend how the physician may misdiagnose the lesion as pelvic cancer. ${ }^{2-3}$ In these cases, biopsy of the endometrium and cervix often reveals extensive inflammatory debris and chronic infection. 


\section{ACKNOWLEDGMENT}

The authors thank David P. Poage, MD, for the computed tomography photographs.

\section{REFERENCES}

1. Monif GRG. Ligneous cellulitis. In: GRG Monif (ed) Infectious Diseases in Obstetrics and Gynecology. 3rd ed. Omaha, NE: IDI Publications, 1994. p 4-109.

2. Campbell C. Ligneous pelvic cellulitis. Pa Med J 1965; $36: 41-42$.

3. Collin CG, Davidson VA, Mathews NM. Use of cortisone in pelvic cellulitis. New Orleans Med Surg J 1952; 104:389-394.
4. Weiland AJ, Tarzy BJ, Young PE. Ligneous cellulitis associated with an IUD. Obstet Gynecol 1978;51:488513.

5. Barnham H, Xerby J, Chandler RS, Hillar MR. Group C streptococci in human infections: A study of 305 isolates with clinical correlations. Epidemiol Infect 1989;102: 379-390.

6. Gossling J. Occurrence and pathogenicity of the Streptococcus milleri group. Rev Infect Dis 1988;10:257-285.

7. Gefland HS, Hodgkiss T, Simmons BP. Multiple hepatic abscesses caused by Streptococcus milleri in association with an intrauterine device. Rev Infect Dis 1989; 11:983-987.

8. Hoffman R, Silverstein MN, Hromas R. Primary thrombocythemia. In Hematology: Basic Principles and Practice. 2nd ed. New York: Churchill Livingstone, 1995. p. 1174-1184. 


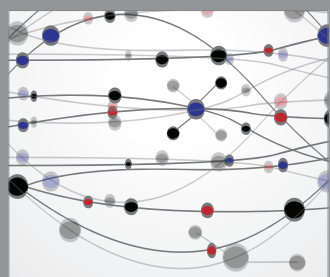

The Scientific World Journal
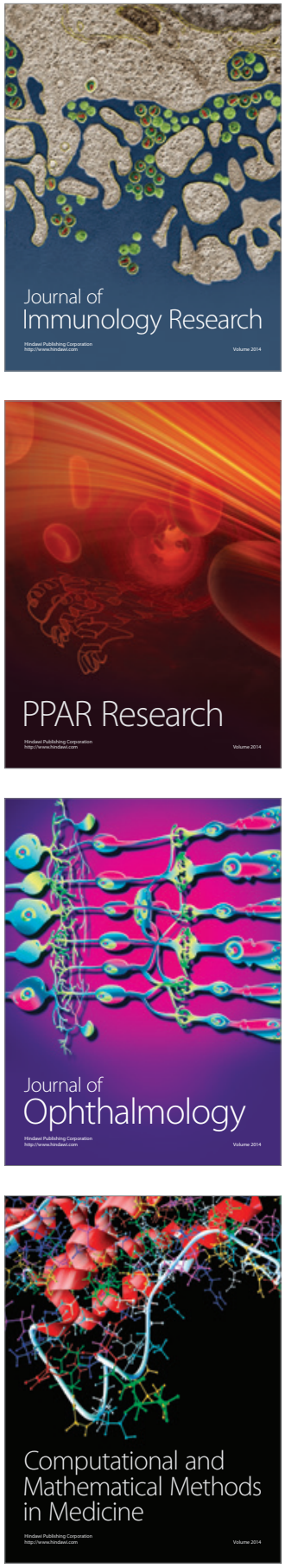

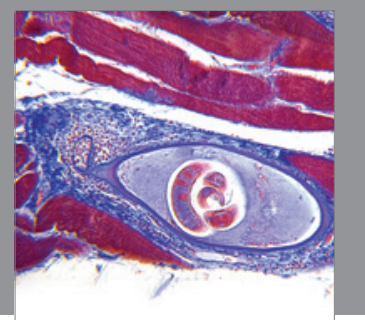

Gastroenterology

Research and Practice
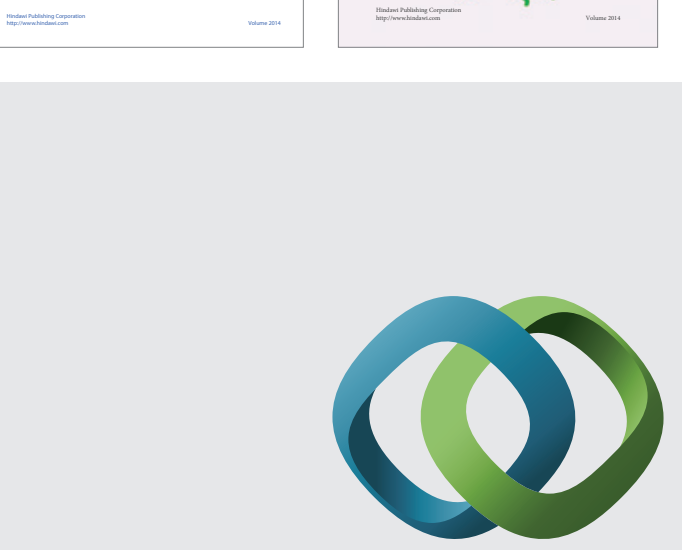

\section{Hindawi}

Submit your manuscripts at

http://www.hindawi.com
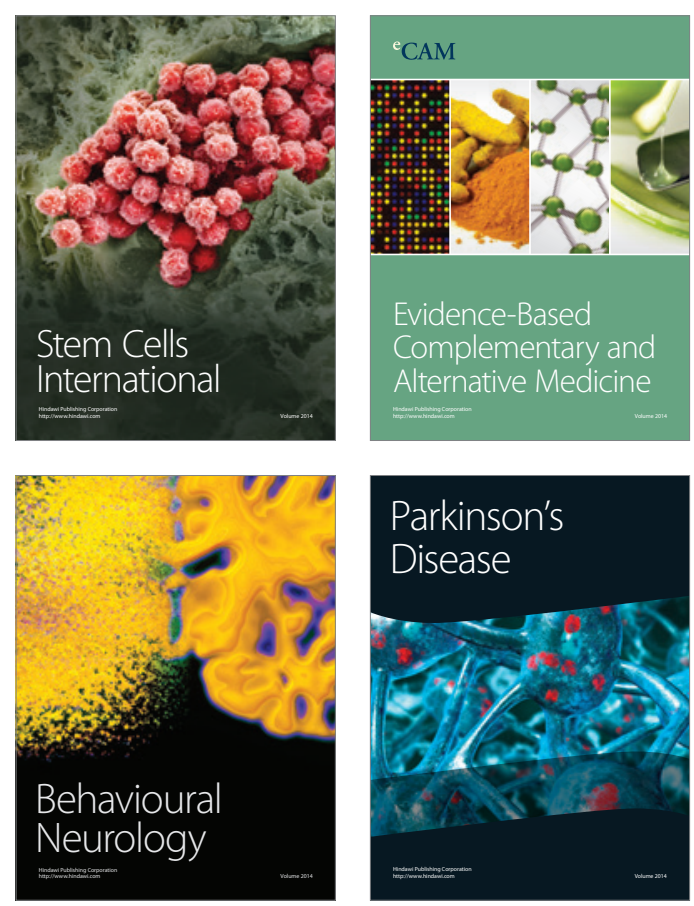

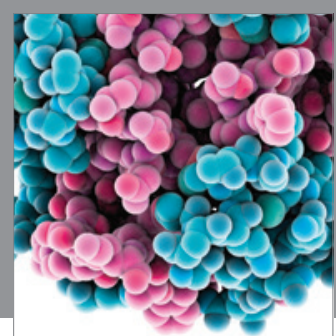

Journal of
Diabetes Research

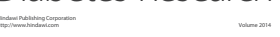

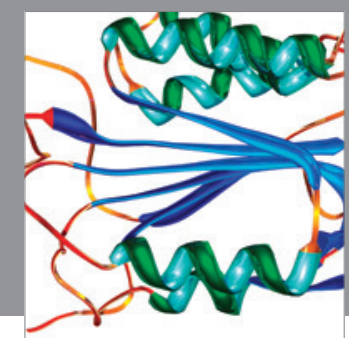

Disease Markers
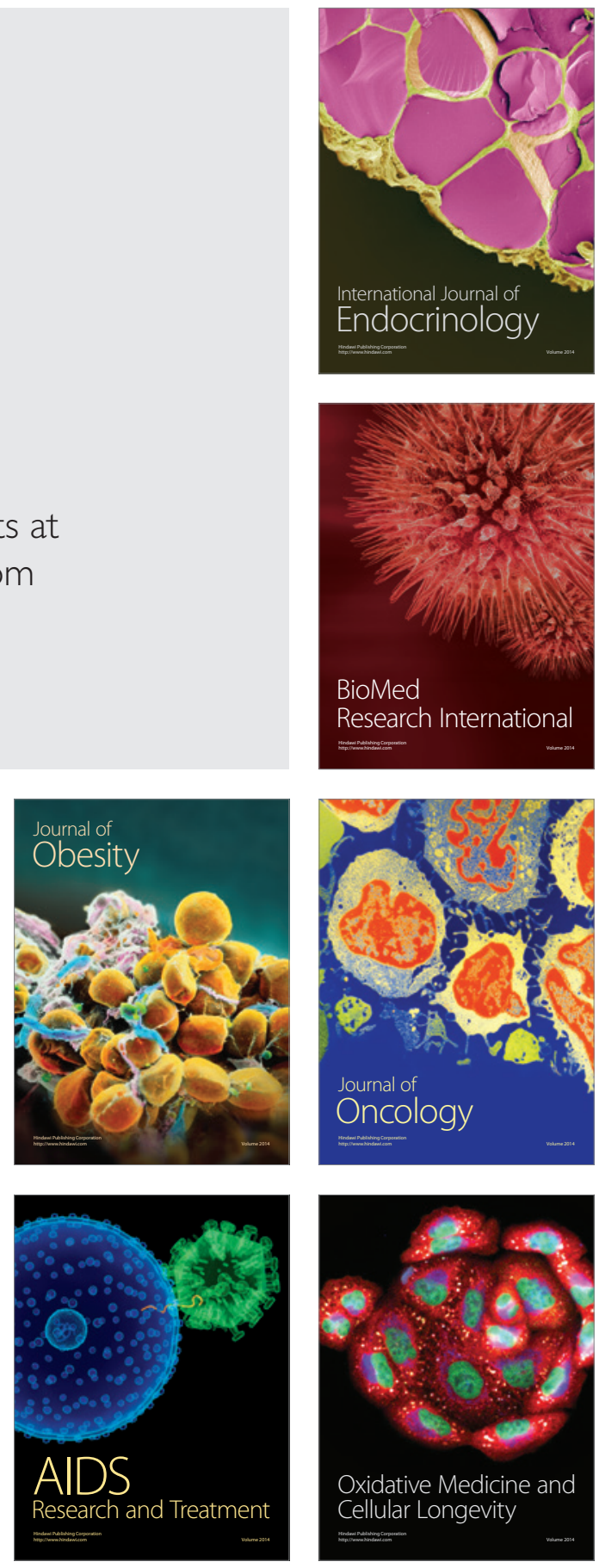